\title{
NUMERICAL STUDIES OF A SECOND HARMONIC RF CAVITY FOR THE IPNS RCS ${ }^{*}$
}

\author{
J. C. Dooling ${ }^{*}$, F. R. Brumwell, G. E. McMichael, M. E. Middendorf, and R. A. Zolecki, \\ ANL, Argonne, IL
}

\begin{abstract}
The Intense Pulsed Neutron Source (IPNS) Rapid Cycling Synchrotron (RCS) delivers $450-\mathrm{MeV}$ protons in $70 \mathrm{~ns}$ pulses at $30 \mathrm{~Hz}$ to a heavy-metal target producing spallation neutrons for material science research. The average current extracted from the RCS is $15 \mu \mathrm{A}$ with a peak intensity of 10 Amps. The large circulating currents in the RCS generate oscillations in the bunch which are presently controlled by modulating the phase of the two rf cavities. By adding second harmonic ( $\mathrm{SH}$ ) rf, the bunch length can be increased reducing the peak current. Simulations suggest that a 20-40 percent increase in extracted current should be achievable. The simulation program allows for phasing between fundamental and $\mathrm{SH}$ rf voltages. Initial studies to optimize phase indicate the need to maximize bucket area early in the acceleration cycle, whereas bunching factor is more significant later in the cycle.
\end{abstract}

\section{INTRODUCTION}

During the 1998 Fiscal Year, the Intense Pulsed Neutron Source (IPNS) conducted 354 experiments and was oversubscribed by almost a factor of two. IPNS is looking at several options to accommodate the demand for neutrons. One option is to increase the neutron flux available from the source by raising the incident proton beam current. Installing a second harmonic (SH) rf cavity in the RCS will increase the current limit of the synchrotron and decrease capture losses. The SH rf cavity should raise proton current to the target by $20-40$ percent. Based upon this increase, IPNS could conduct an additional 100 experiments annually. Alternatively, higher current would improve the signal-to-noise ratio in collected data or provide better resolution for experiments.

SH rf was first proposed for the IPNS in the early 1980's but not pursued due to funding constraints and loss of personnel[1]. Elsewhere, ISIS is preparing to install a SH system which is expected to result in a 50 percent increase in current from their RCS[2,3].

\section{MODELING}

Several approaches are used to model capture and acceleration in the RCS. A longitudinal particle dynamics code, CAPTURE_SPC $[4,5]$ (CAPT) is employed to study trapping and loss of injected beam including the effects of space charge. A predictor-corrector algorithm is used to independently compare phase-space area to that calculated with CAPT for the case of no space charge. Both analyses yield essentially identical results for the bucket phasespace area. The evolution of RCS bucket size during the acceleration cycle is shown in Figure 1.

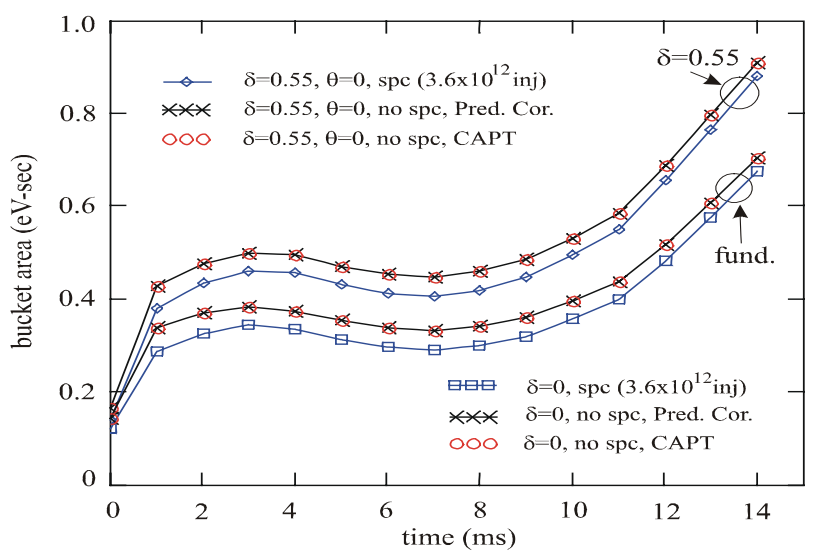

Figure 1: Evolution of stable phase-space in the RCS showing the effect of SH rf and space-charge

Longitudinal equations of motion for energy and phase may be written as,

$$
\begin{aligned}
& \frac{\mathrm{d}}{\mathrm{dt}}\left[\frac{\Delta \mathrm{E}}{\omega_{\mathrm{o}}}\right]=\frac{\mathrm{q}}{2 \pi}\left[\mathrm{V}(\phi, \theta)-\mathrm{V}\left(\phi_{\mathrm{s}}, \theta\right)+\mathrm{V}_{\mathrm{sc}}(\phi)\right] \\
& \frac{\mathrm{d}}{\mathrm{dt}} \Delta \phi=\frac{\mathrm{h} \omega_{\mathrm{o}} \eta}{\beta^{2} \mathrm{E}_{\mathrm{s}}} \Delta \mathrm{E}_{\mathrm{s}}
\end{aligned}
$$

where $\mathrm{q}$ is the charge, $\mathrm{V}$ is the amplitude of the rf voltage, $\phi$ is the phase of the bunch with respect to the rf, $h$ is the harmonic number ( $h=1$, in the IPNS RCS), $\omega_{0}$ is the cyclotron frequency, $\eta$ is the slip factor, $\beta=v / c, E$ is the total energy of the particle, and the subscript s refers to the synchronous particle. In the RCS $\beta$ varies from 0.316 at injection to 0.737 at extraction corresponding to a frequency range of 2.21-5.14 MHz. Allowing for the presence of a second harmonic,

\footnotetext{
*Work supported by U.S. DOE, Contract W-31-109-ENG-38

*Email: jcdooling@anl.gov
} 


$$
\mathrm{V}(\phi, \theta)=\mathrm{V}[\sin (\phi)-\delta \sin (2 \phi+\theta)]
$$

where $\delta$ is the ratio of second to first harmonic amplitudes and $\theta$ is phase shift. The space charge term may be expressed as,

$$
\mathrm{V}_{\mathrm{sc}}=-\mathrm{q} \frac{\mathrm{d} \lambda(\phi)}{\mathrm{d} \phi}\left[\frac{\mathrm{Rg}}{2 \varepsilon_{\mathrm{o}} \gamma^{2}}-\mathrm{L} \beta^{2} \mathrm{c}^{2}\right] \frac{1}{\mathrm{R}^{2}}
$$

where $\lambda$ is line density, $g_{o}$ is the capacitive coupling factor, and L is total inductance per turn. CAPT tracks particles using a leap-frog algorithm to achieve secondorder accuracy. Figure 2 presents phase-space and separatrix results for the RCS with fundamental-only rf, 8 $\mathrm{ms}$ after injection. A gaussian energy spread is assumed at injection with $\Delta \mathrm{p} / \mathrm{p}=0.3$ percent, FWHM.
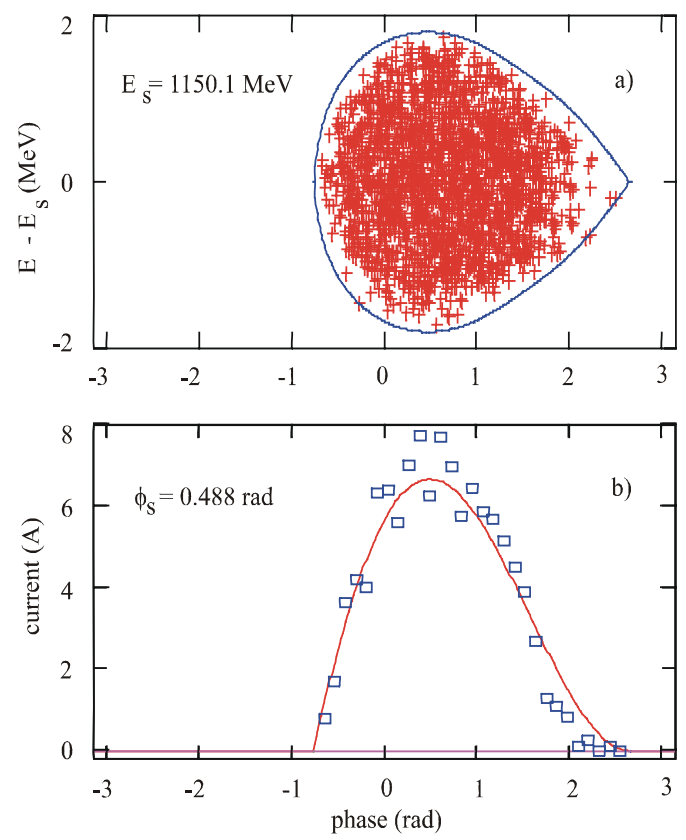

Figure 2: a) Bunch and phase-space from CAPT and b) current profiles from CAPT ( $\square$ ) and HP (-) for fundamental-only rf at $\mathrm{t}=8 \mathrm{~ms}$.

\subsection{Bunch Factor}

With proper phasing, $\mathrm{SH}$ rf raises the bunch factor (BF) by flattening the longitudinal current density profile. Reducing the peak current density is one method of delaying the onset of current-driven instabilities[6,7]. BF is defined as,

$$
\mathrm{BF}=\frac{1}{2 \pi} \int_{\phi_{1}}^{\phi_{2}} \frac{\mathrm{I}(\phi)}{\mathrm{I}_{\max }} \mathrm{d} \phi
$$

The original implementation of CAPT has been modified to allow for variation in $\theta$ to optimize BF. In Figure 3, CAPT results are presented with $\delta=0.55$ and $\theta=-1.072 \mathrm{rad}$, which maximizes $\mathrm{BF}$ at 0.487 ; for the fundamental-only case shown in Fig. 2, BF=0.306. In both cases, the same rf voltage program is used.
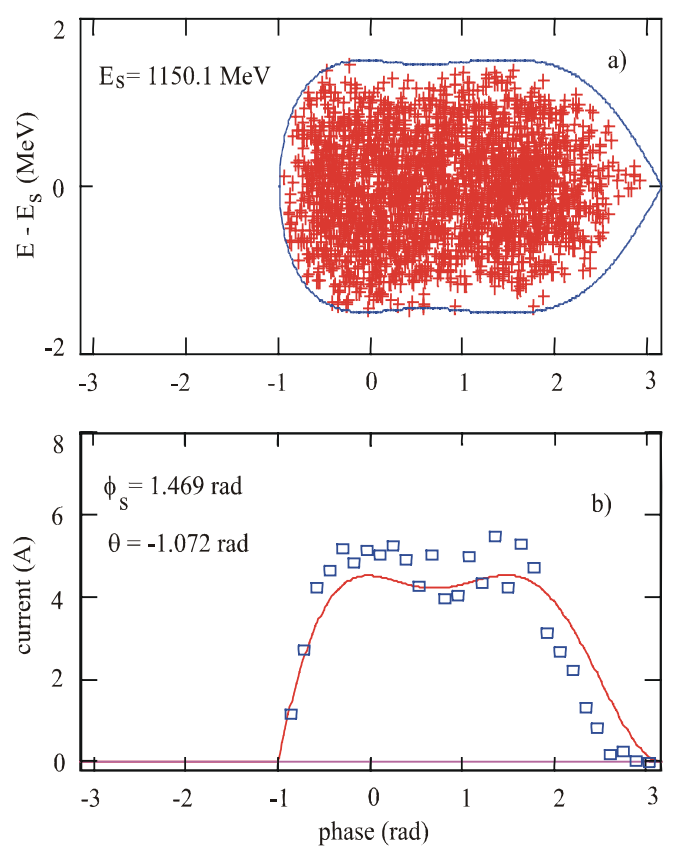

Figure 3: Same as Fig. 2 but with $\mathrm{SH}(\delta=0.55)$ and $\theta$ to maximize $\mathrm{BF}$.

\section{$2.2 \theta$ and Losses}

Modifying $\theta$ can help reduce acceleration losses. For the IPNS rf voltage program, the bucket tends to shrink until reaching a minimum near $\dot{\mathrm{B}}$ max . CAPT predicts that after the initial trapping loss, particles continue to leave the bucket as the bunch is accelerated. The bunch fills the entire available phase-space after capture; therefore, any reduction in the bucket size will result in losses. The reduction in bucket size between 3 and $7 \mathrm{~ms}$ is evident in Fig. 1. For a given $\delta$, maximizing BF during the acceleration cycle by varying $\theta$, results in a smaller stable phase-space area (bucket size) than with $\theta=0$. The resulting loss can be seen in Figures 4 a) and b). Note that overall efficiency is improved relative to the $\theta$ profile where $\mathrm{BF}$ is maximized $\left(\theta_{\mathrm{m}}\right)$. However, to increase the current limit of the machine, $\theta$ should be varied to increase the BF. The simulation shows that it should be possible to adjust $\theta$ to maximize bucket size early in the cycle, then increase the BF to reduce the peak currents during the later stages of acceleration. One possible strategy is presented in Figure 5. The SH phase angle is kept at 0 until $\dot{\mathrm{B}}$ max $(8 \mathrm{~ms}$, just after the minimum bucket size), then ramped to $\theta_{\mathrm{m}}$ at $\mathrm{t}=10 \mathrm{~ms}$. The $\mathrm{BF}$ is brought to its maximum value after $\dot{\mathrm{B}} \max _{\text {ax }}$, where stable phasespace is increasing with time and, according to the analysis, acceleration losses cease. 


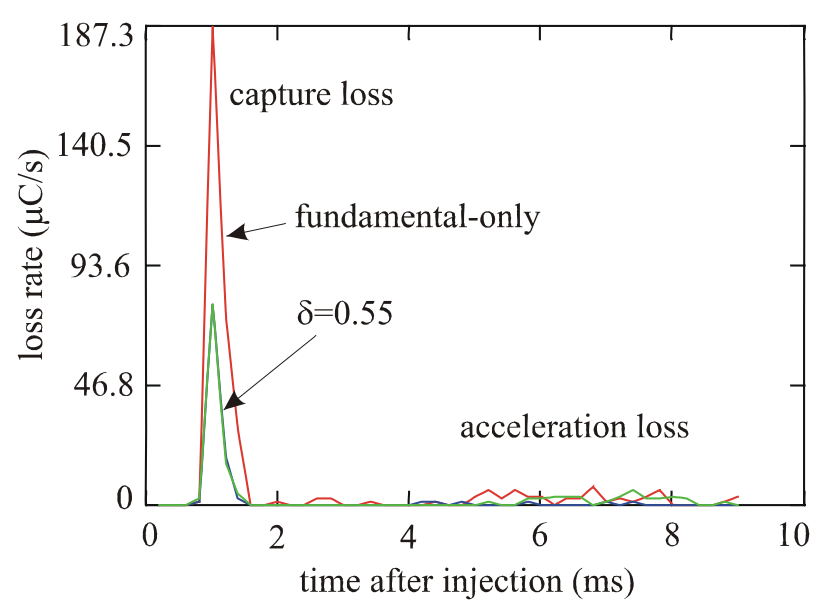

Figure 4a: Capture and acceleration losses

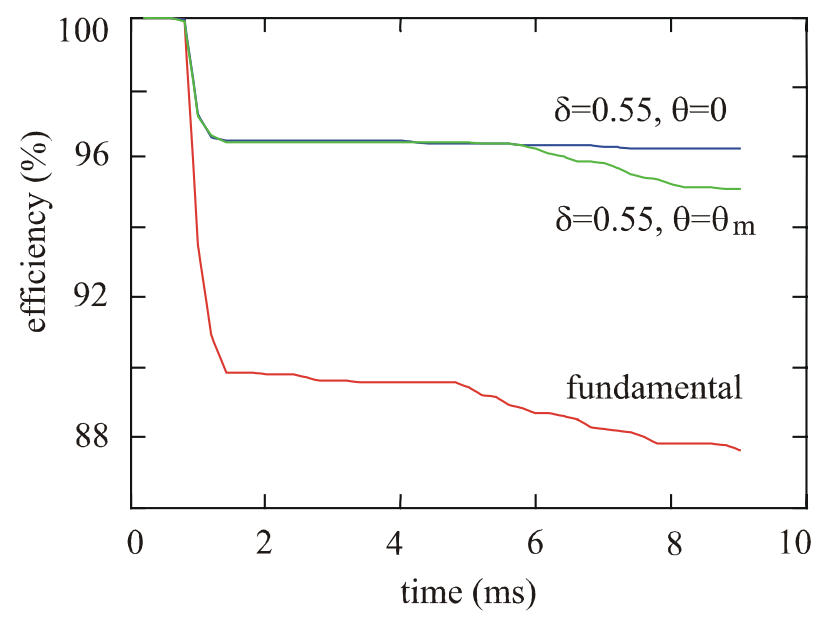

Figure 4b: Integrated loss rate for three rf cases: $\delta=0.55$, $\theta=0 ; \delta=0.55, \theta=\theta_{\mathrm{m}} ;$ and fundamental rf only.

\section{CURRENT LIMITS}

Hoffman-Pedersen (HP) distributions[7] are used to examine current limits in the RCS. HP current profiles are shown in Figures 2 and 3 along with CAPT results. For the case of fundamental-only rf, peak current values at $8 \mathrm{~ms}$ are $6.7 \mathrm{~A}$ and $7.1 \mathrm{~A}$ from $\mathrm{HP}$ and CAPT, respectively; using $\mathrm{SH}$ rf with amplitude and phase as shown in Fig. 3, the peak currents are 4.5 A and 5.1 A. HP analysis indicates a microwave instability current limit for the machine in the range of 10-11 A. In the fundamental case just prior to extraction, peak current has risen to $10.8 \mathrm{~A}$ according to CAPT, in agreement with current-toroid measurements. With phased $\mathrm{SH}$ rf, near extraction time, calculations with CAPT predict a peak current of 7.8 A for the same injected charge.

Presently, the IPNS RCS is operating close to its current limit. By introducing SH rf with a third cavity, it should be possible to raise the current delivered to the target by $20-40$ percent.

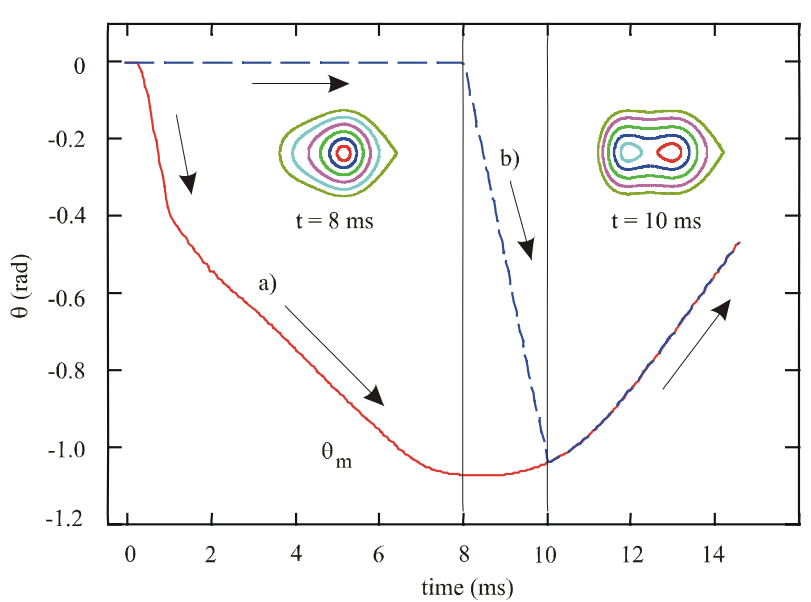

Figure 5: Phasing of the $\mathrm{SH}$ rf for a) maximum $\mathrm{BF}$ and b) better transmission $(\delta=0.55)$. Phase-space contours before and after the ramp are also shown.

\section{REFERENCES}

[1] J. Norem, F. Brandeberry, and A. Rauchas, IEEE Trans. Nuc. Sci., 30(4), 3490(1983). .

[2] C. R. Prior, Proc. of the Twelfth Meeting of the International Collaboration on Advanced Neutron Sources, Abingdon, May 1993, RAL 94-025, Vol. II, p. A-11.

[3] M. R. Harold, M. G. Glover, I. S. K. Gardner, and R. G. Bendal, "A Proposal to Improve the Performance of ISIS," ISIS Management Committee Report IMC/P16/96, 1996.

[4] Y. Cho, E. Lessner, K. Symon, Proc. European Particle Accelerator Conf., p. 1228, (1994).

[5] E. Lessner and K. Symon, Computational Accelerators Physics, Williamsburg, Virginia, September 1996, AIP Conf. Proc. 391, p.185 (1997).

[6] P. Bramham, S. Hansen, A. Hofmann, E. Peschardt, IEEE Trans. Nuc. Sci., 24(3), 1490(1977).

[7] A. Hofmann and F. Pedersen, IEEE Trans. Nuc. Sci., 26(3), 3526 (1979). 